\title{
Solar flux dependence of coherence scales in scintillation patterns produced by ESF irregularities
}

\author{
B. Engavale, K. Jeeva, K.U. Nair, and A. Bhattacharyya \\ Indian Institute of Geomagnetism, Kalamboli Highway, Kalamboli - 410218 New Panvel, India
}

Received: 24 February 2005 - Revised: 5 September 2005 - Accepted: 20 September 2005 - Published: 30 November 2005

\begin{abstract}
The coherence scale length, defined as the 50\% decorrelation scale length along the magnetic east-west direction, in the ground scintillation pattern obtained at a dip equatorial location, due to scattering of VHF radio waves by equatorial spread $\mathrm{F}$ (ESF) irregularities, is calculated, using amplitude scintillation data recorded by two spaced receivers. The average east-west drift of the ground scintillation pattern, during the pre- and post-midnight periods, also calculated from the same observations, shows an almost linear increase with 10.7-cm solar flux. In the present paper the variability of the drift is automatically taken into account in the calculation of the coherence scale length of the ground scintillation pattern. For weak scintillations, the coherence scale depends on the Fresnel scale, which varies with the height of the irregularity layer, and also on the spectral index of the irregularity power spectrum. It is found that for weak scintillations, the coherence scales are much better organized according to the $10.7-\mathrm{cm}$ solar flux, during the pre-midnight period, than during the post-midnight period, with a general trend of coherence scale length increasing with $10.7-\mathrm{cm}$ solar flux except for cases with F 10.7-cm solar flux $<100$. This indicates that, during the initial phase of ESF irregularity development, the irregularity spectrum does not have much variability while further evolution of the spatial structure in ESF irregularities is controlled by factors other than the solar flux.
\end{abstract}

Keywords. Ionosphere (Equatorial ionosphere; Ionospheric irregularities; Plasma waves and instabilities)

\section{Introduction}

The generation and evolution of equatorial spread $\mathrm{F}$ (ESF) irregularities through plasma instabilities (Haerendel, 1973; Ossakow, 1981; Keskinen et al., 1998, 2003; Bhattacharyya, 2004) depends on ambient ionospheric conditions present in

Correspondence to: A. Bhattacharyya

(archana@iigs.iigm.res.in) the equatorial and low-latitude regions. Hence, day-to-day variability of the ionosphere in these regions is reflected in the day-to-day variations in the occurrence and evolution of ESF irregularities. In a large number of studies using ionosondes, HF Doppler, and VHF radars, the height of the nighttime equatorial F-layer has emerged as the most important ionospheric parameter to control the generation of ESF irregularities (Farley et al., 1970; Abdu et al., 1983; Sastri, 1984; Kelley and Maruyama, 1992; Jayachandran et al., 1993). Further, on the basis of long-term radar observations carried out at the Jicamarca Observatory, Fejer et al. (1999) have found that the threshold value for vertical plasma drift velocity for the generation of strong early night irregularities increases linearly with solar flux. In the present paper, ionospheric scintillation data is used to study the dependence of the spatial structure of ESF irregularities on solar flux during the phase when the irregularities produce weak scintillations. It is well known that ESF irregularities span a large range of scale sizes, extending over many orders of magnitude. Of these, irregularities with scale sizes in the intermediate range, which extend from tens of kilometers down to a few hundred meters, give rise to a forward scattering of VHF or higher frequency radio waves, transmitted from a geostationary satellite and recorded on the ground. The resultant diffraction pattern on the ground drifts past a ground receiver, due to the drift of the irregularities across the signal path, thus converting spatial variations of intensity into temporal variations or scintillations, recorded by a ground receiver. The irregularities evolve as they drift, and this causes random temporal fluctuations of the received signal. The ESF irregularity drift also changes through the night. Therefore, extraction of information about spatial scales present in the ground diffraction pattern, which are related to various characteristics of the irregularities including height from scintillation data is not a straightforward exercise.

Recently, a method has been devised for determining the spatial scales present in the ground scintillation pattern from scintillation data recorded by two spaced receivers (Bhattacharyya et al., 2003). In Sect. 2, this method is used to 


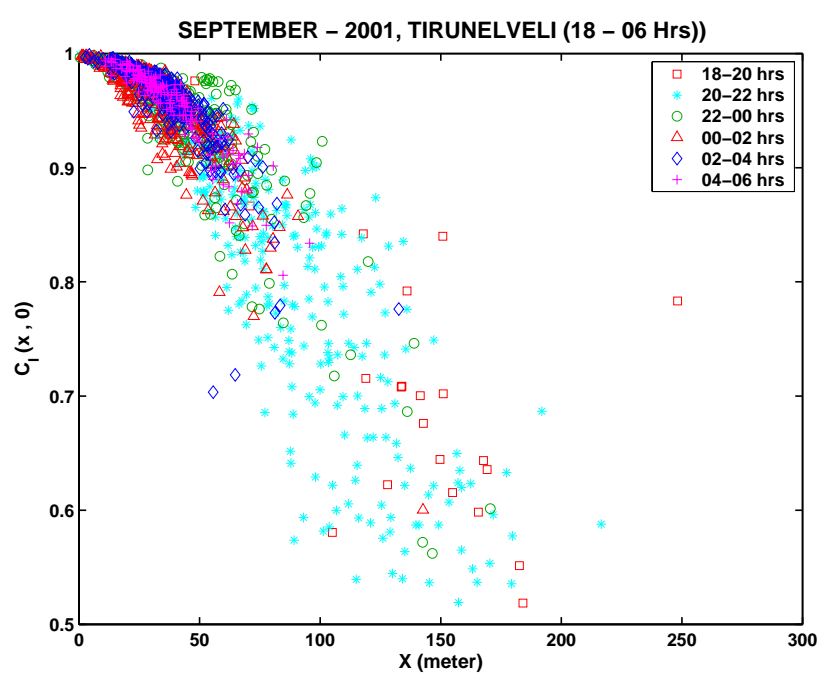

Fig. 1. Mass plot of $C_{I}(x, 0)$ as a function of $x$ for the month of September 2001. The points are divided into 2-h bins representing different stages of evolution of the irregularities shown by different symbols.

estimate the distribution of spatial scale sizes present in the ground diffraction pattern for periods of weak scintillations during a scintillation event. The method itself is also applicable to strong scintillations. The reason for restricting the study here to weak scintillations is discussed in Sect. 3, where the distribution of scale sizes is studied as a function of solar flux. Dependence of the corresponding zonal irregularity drift, calculated from the data, on solar flux is also described in this section. Results obtained are discussed in Sect. 4 and the conclusions drawn from these results are presented in Sect. 5.

\section{Data analysis}

The data used in the present study consists of amplitude scintillations on a $251-\mathrm{MHz}$ signal transmitted from the geostationary satellite, UFO2, located at $71.2^{\circ} \mathrm{E}$, and recorded by two spaced receivers at the equatorial station Tirunelveli (dip latitude $0.6^{\circ} \mathrm{N}$ ) during the period June 2001 to April 2004. In addition, amplitude scintillations on a $251-\mathrm{MHz}$ signal transmitted from the geostationary satellite, FLEETSAT, located at $73^{\circ} \mathrm{E}$ and recorded at Tirunelveli, for the equinoctial months of 1992, 1996, 1998 to 2000 have been included in the study. Since ESF irregularities have been found to be closely aligned with the geomagnetic field (Tsunoda, 1980) spatial variations of intensity in the ground scintillation pattern at Tirunelveli are expected to be in the east-west direction. Hence, the receivers are placed along an east-west baseline with a separation of $540 \mathrm{~m}$, which is comparable to the Fresnel scale. In order to estimate the average eastward drift speed $V_{0}$ of the ground scintillation pattern, when random temporal changes in irregularity characteristics are present (Wernik et al., 1983), a full correlation analysis technique first suggested by Briggs (1984) is used. The basis of this technique is the assumption that the space-time correlation function of intensity in the ground pattern, for variations in one dimension, is of the form:

$C_{I}(x, t)=f\left[\left(x-V_{0} t\right)^{2}+V_{C}^{2} t^{2}\right]$,

where $f$ is a monotonically decreasing function of its argument, with $f(0)=1$, and the parameter $V_{C}$, generally referred to as the random velocity, is introduced, to take into account the decorrelation due to random temporal variations in the pattern which is drifting along the $x$-direction with an average speed $V_{0}$. Estimation of $V_{0}$ and $V_{C}$ from spaced receiver data does not require a knowledge of the exact form of the function $f$ (Vacchione et al., 1987; Spatz et al., 1988; Bhattacharyya et al., 1989). However, in order to obtain information about spatial scales present in the ground scintillation pattern, it is necessary to know the form of $f$, such that the coherence scale $d_{I}$, defined by

$C_{I}\left(x=d_{I}, t=0\right)=0.5$,

which gives an estimate of the dominant spatial scale present in the ground scintillation pattern, may be calculated from the data.

An examination of Eq. (1) shows that for two receivers separated by a distance $x_{0}$, the maximum value of the crosscorrelation function of signals recorded by them occurs at a time lag $t_{m}$ which depends on the values of $x_{0}, V_{0}$ and $V_{C}$, and this value is less than one on account of decorrelation. This value is, in fact, given by Bhattacharyya et al. (2003):

$C_{I}\left(x_{0}, t_{m}\right)=f\left[\frac{x_{0}^{2} V_{C}^{2}}{V_{0}^{2}+V_{C}^{2}}\right]$.

Values of $C_{I}\left(x_{0}, t_{m}\right), V_{C}$ and $V_{0}$ are estimated from spaced receiver data. As the ESF irregularities evolve, variations in their spatial structure, the height and strength of perturbations in density and the electric field associated with them, produce different sets of values of these parameters. A mass plot of $C_{I}\left(x_{0}, t_{m}\right)$ versus $x_{0} V_{C} /\left(V_{0}^{2}+V_{C}^{2}\right)^{1 / 2}$ for scintillation events which have been produced by ESF irregularities during post-sunset hours of magnetically quiet days in a month, such as the one displayed in Fig. 1, shows how the functional form of $C_{I}(x, 0)=f\left(x^{2}\right)$ changes at different stages of evolution of ESF irregularities. Variation in the functional form of $C_{I}(x, 0)$ determines the distribution of dominant spatial scale size $d_{I}$ in the ground scintillation pattern as the ESF irregularities evolve.

The assumption about the form of the space-time correlation function inherent in Eq. (1) requires that the estimation of $V_{0}$ and $V_{C}$ from spaced receiver scintillation data be restricted to only those data intervals which have $C_{I}\left(x_{0}, t_{m}\right) \geq 0.5$, because in this situation the assumption is likely to have greater validity. Also, in order to reduce the effects of noise in the data, estimation of these parameters has been carried out only when the $S_{4}$-index, which is the standard deviation of normalized intensity fluctuations and thus a measure of the strength of scintillations, is not less 


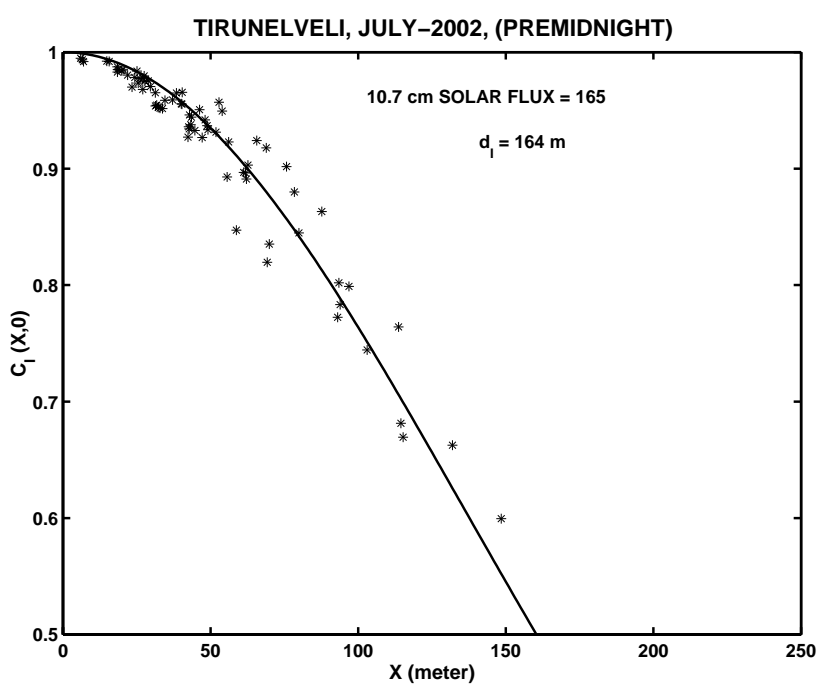

Fig. 2. $C_{I}(x, 0)$ as a function of $x$ for the pre-midnight period of weak scintillations during July 2002.

than 0.15 . Whenever these conditions are satisfied, $V_{0}, V_{C}$ and $C_{I}\left(x_{0}, t_{m}\right)$ have been estimated for each period of $3 \mathrm{~min}$ during a scintillation event, from data sampled at intervals of $0.1 \mathrm{~s}$. The background ionospheric conditions show a great deal of variability from one month to another, which would give rise to different patterns of evolution of the irregularities during these periods. This is expected to yield different distributions of spatial scale sizes in the ground scintillation pattern in different seasons. In the present study, in order to understand the roles played by various factors which control the characteristics of ESF irregularities, mass plots of $C_{I}\left(x_{0}, t_{m}\right)$ versus $x_{0} V_{C} /\left(V_{0}^{2}+V_{C}^{2}\right)^{1 / 2}$, which are equivalent to mass plots of $C_{I}(x, 0)$ as a function of $x$, are made from estimates of $C_{I}\left(x_{0}, t_{m}\right), V_{0}$ and $V_{C}$ for a period of one month at a time.

\section{Weak scintillation result}

Scintillations due to ESF irregularities are generally expected to be weak $\left(S_{4} \leq 0.25\right)$ when the irregularities just start growing and also when there has been considerable erosion of density fluctuations in the decay phase of irregularities. At other times, during the evolution of irregularities, often scintillations are not in the "weak" category. The reason for restricting the present study to weak scintillations is that, in this case it is relatively straightforward to relate the estimated coherence scale, $d_{I}$, with irregularity characteristics. In fact, theoretical considerations show that the maximum contribution to weak amplitude scintillations is expected to come from irregularities which have scale sizes of the order of the Fresnel scale, and in this situation, the dominant spatial scale present in the ground scintillation pattern is determined by the Fresnel scale (Yeh and Liu, 1982). Theoretical calculations of $d_{I}$ for weak scintillations produced by ionospheric irregularities with a power-law spectrum also show a depen-

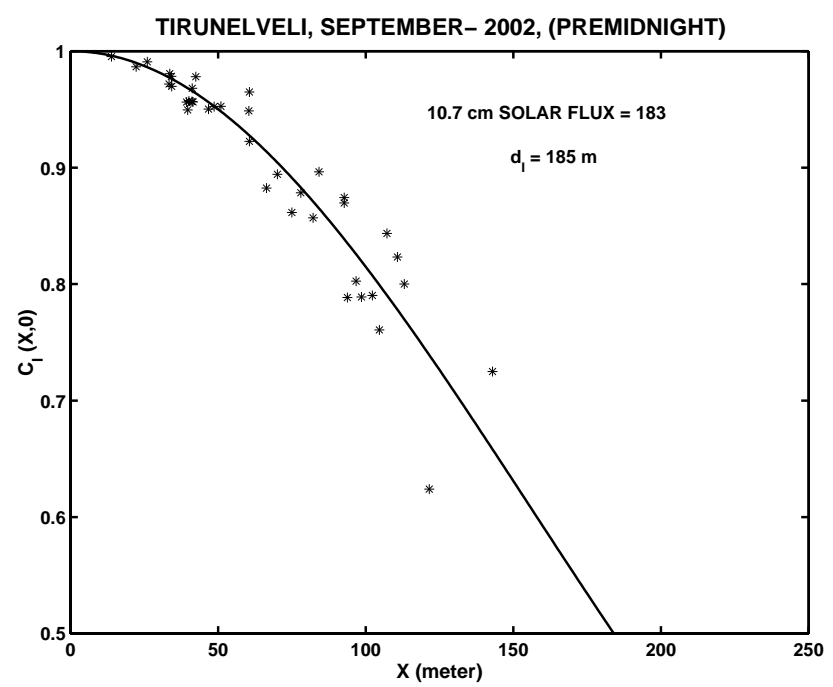

Fig. 3. $C_{I}(x, 0)$ as a function of $x$ for the pre-midnight period of weak scintillations during September 2002.

dence of $d_{I}$ on the power spectral index, both for a phase changing screen (Rino and Owen, 1980) and a thick layer of irregularities (Engavale and Bhattacharyya, 2005). The Fresnel scale is given by $d_{F}=\sqrt{2 \lambda Z_{R}}$, where $\lambda$ is the signal wavelength and $Z_{R}$ is the average distance of the irregularity layer from the receiver, which would be determined by the average height of the irregularity layer for a fixed signal path, as in the case of a geostationary satellite.

In scintillation data, spatial scales in the ground scintillation pattern are converted into temporal scales due to the drift of the irregularities across the signal path. The velocity with which irregularities drift across the signal path changes throughout the course of a scintillation event. In the first few hours of ESF irregularity development, values of $V_{0}$ obtained from spaced receiver scintillation data show a great deal of variability, since during this phase, perturbation electric fields associated with the Rayleigh-Taylor (R-T) instability, which gives rise to the scintillation producing ESF irregularities, produce $\boldsymbol{E} \times \boldsymbol{B}$ drift of the irregularities (Bhattacharyya et al., 2001). On magnetically quiet days, after about 22:00 LT, the irregularities are found to drift with the background plasma. In the method used here for estimation of the spatial coherence scale $d_{I}$ (Bhattacharyya et al., 2003), variations in $V_{0}$ are automatically taken into account in determination of the spatial correlation function $C_{I}(x, 0)$. Average height of the irrgularity layer varies during the course of a scintillation event, and may be markedly different during preand post-midnight periods. It is also expected that power-law irregularity spectra would have different spectral indices during pre- and post-midnight periods, with faster decay of short scale length irregularities in the later period. Hence, separate mass plots of $C_{I}\left(x_{0}, t_{m}\right)$ versus $x_{0} V_{C} /\left(V_{0}^{2}+V_{C}^{2}\right)^{1 / 2}$ are made for these two periods.

The average eastward drift of equatorial F-region plasma during pre-midnight periods of scintillation events is 


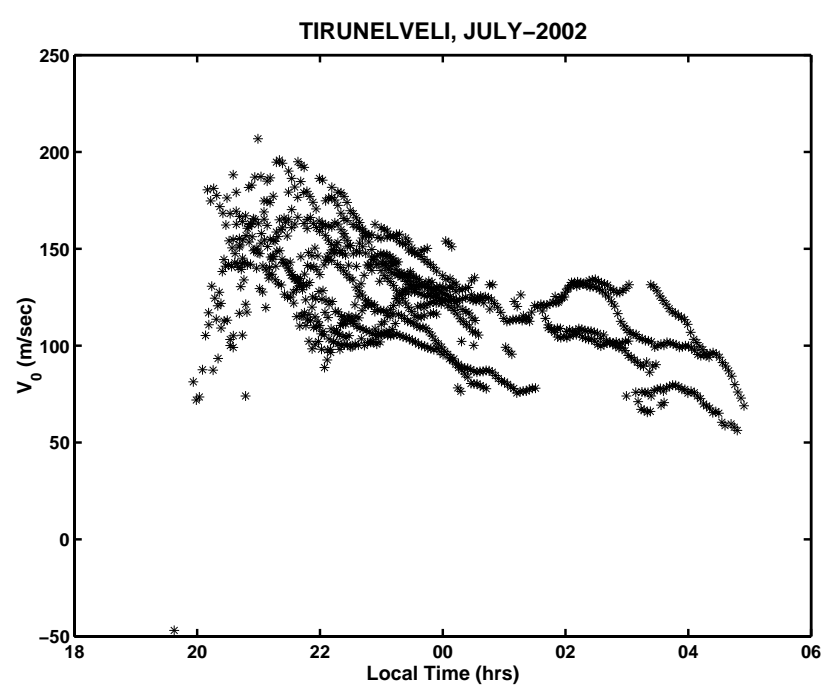

Fig. 4. Mass plot of average eastward drift, $V_{0}$, of the ground scintillation pattern as a function of local time for July 2002.

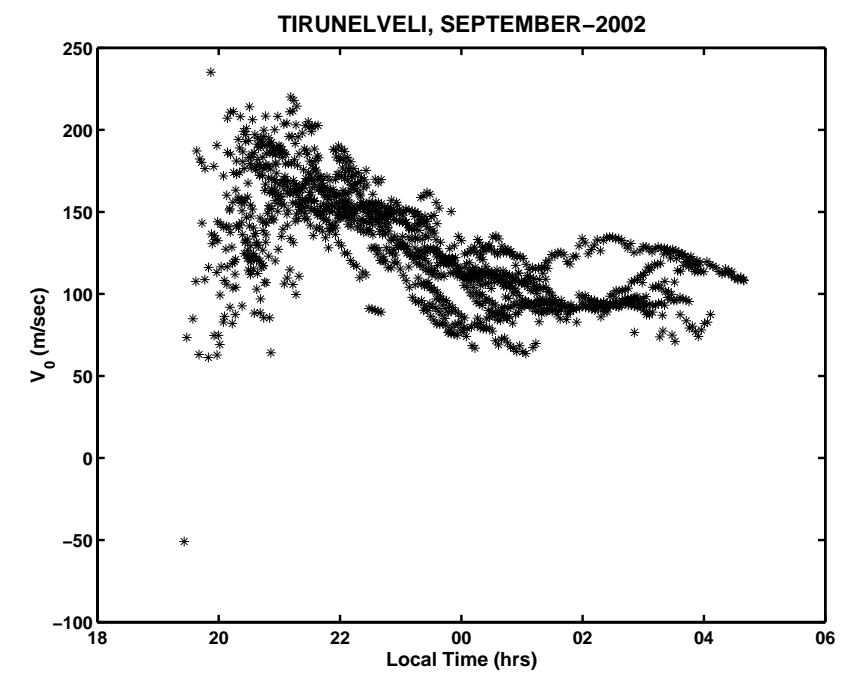

Fig. 5. Mass plot of average eastward drift, $V_{0}$, of the ground scintillation pattern as a function of local time for September 2002.

obtained by considering the estimated $V_{0}$ values for local times later than 22:00 LT, when, as stated above, the ESF irregularities simply, drift along with the background plasma (Bhattacharyya et al., 2001). For obtaining a monthly representative value of $d_{I}$ for pre- and post-midnight periods of weak scintillations, the mass plots of $C_{I}(x, 0)$ versus $x$ for such periods are fitted by Gaussians: $C_{I}(x, 0)=e^{-\left(x / d_{0}\right)^{2}}$ using a least-squares method to calculate $d_{I}=0.832 d_{0}$. Two examples of mass plots of $C_{I}(x, 0)$ versus $x$ for the premidnight weak scintillation periods during the months of July 2002 and September 2002, are shown in Figs. 2 and 3. For these two months, mass plots of $V_{0}$ as a function of local time are displayed in Figs. 4 and 5, respectively. Coherence scales $d_{I}$, calculated for pre- and post-midnight periods of each month, are plotted against the average $10.7-\mathrm{cm}$ solar

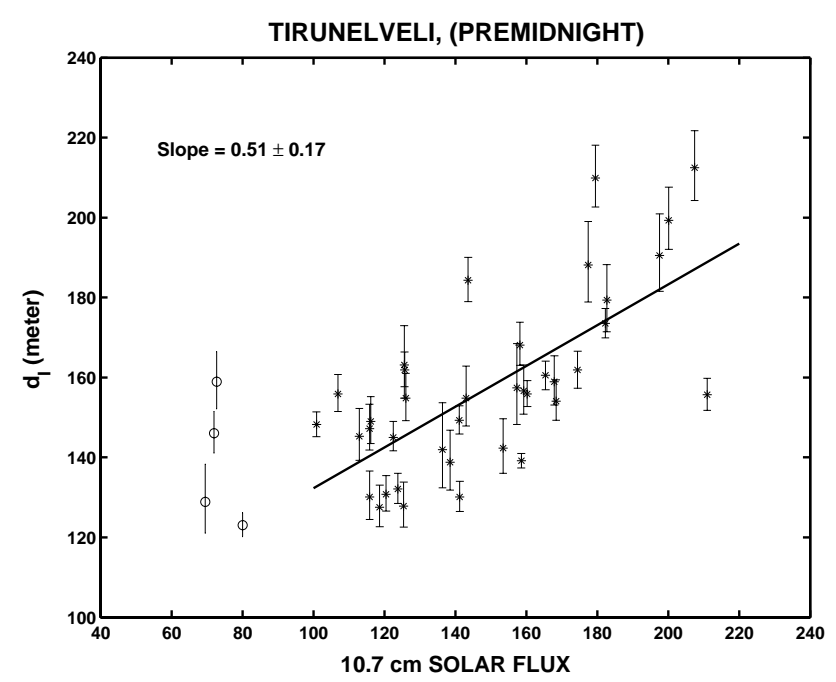

Fig. 6. Variation of coherence scale $d_{I}$, for pre-midnight weak scintillation patterns, with monthly average $10.7-\mathrm{cm}$ solar flux (in units of $10^{-22} \mathrm{~W} \mathrm{~m}^{-2} \mathrm{~Hz}^{-1}$ ).

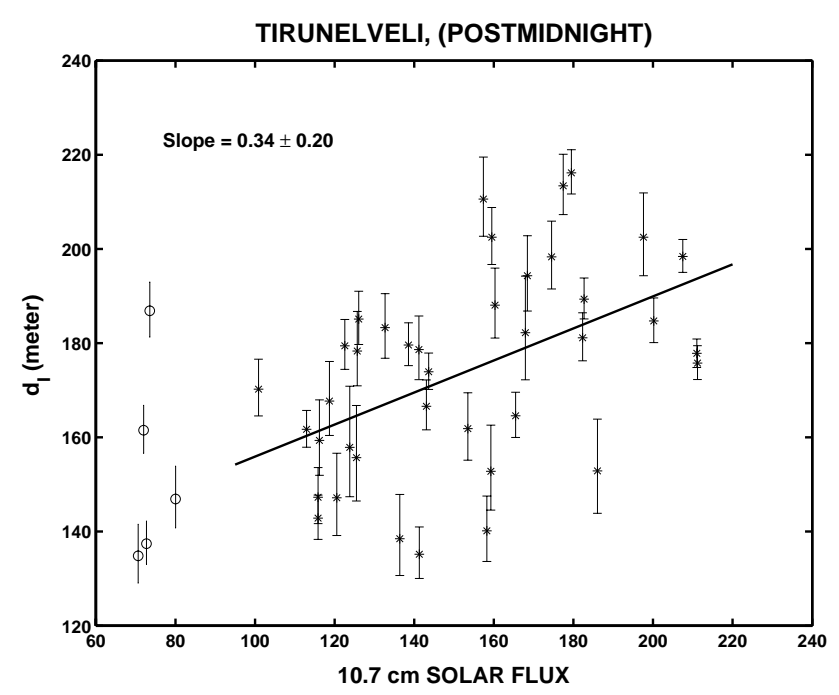

Fig. 7. Variation of coherence scale $d_{I}$, for post-midnight weak scintillation patterns, with monthly average $10.7-\mathrm{cm}$ solar flux (in units of $10^{-22} \mathrm{~W} \mathrm{~m}^{-2} \mathrm{~Hz}^{-1}$ ).

flux (in units of $10^{-22} \mathrm{~m}^{-2} \mathrm{~Hz}^{-1}$ ) for days with scintillations during the month, in Figs. 6 and 7, respectively. Monthly average values of $V_{0}$ during pre- and post-midnight periods are also plotted as functions of the 10.7-cm solar flux in Figs. 8 and 9 , respectively.

\section{Discussion}

For the signal path from the geostationary satellite UFO2 (FLEETSAT), to the receiver at Tirunelveli, zenith angle $\theta=12.8^{\circ}\left(11.4^{\circ}\right)$, and the azimuth is $\phi=216.9^{\circ}\left(105.6^{\circ}\right)$. Hence, both the vertical drift $V_{Z}$ and eastward drift $V_{E}$, of the irregularities, contribute to $V_{0}=V_{E}-V_{Z} \tan \theta \sin \phi$. The 


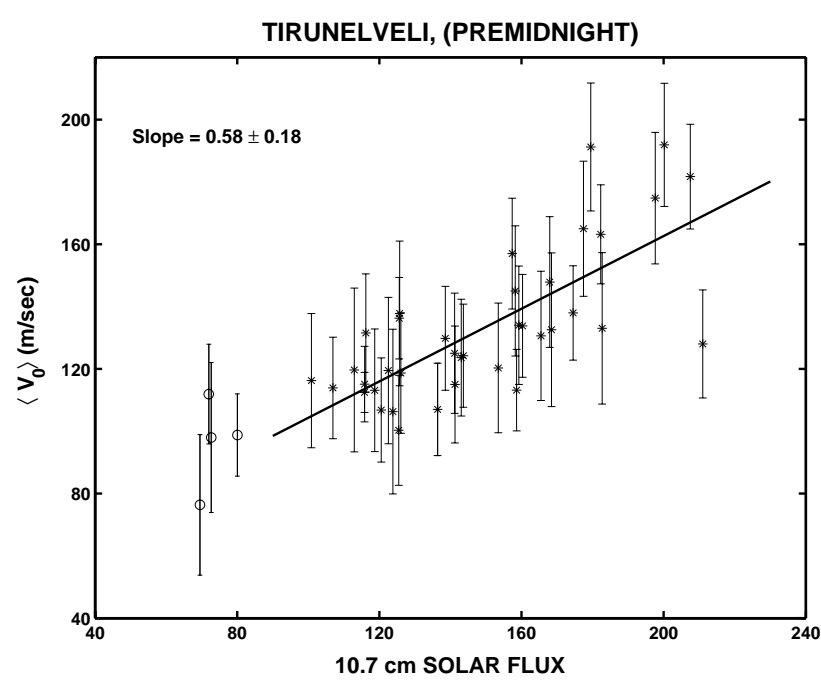

Fig. 8. Plot of average $V_{0}$, for pre-midnight hours versus monthly average $10.7-\mathrm{cm}$ solar flux (in units of $10^{-22} \mathrm{~W} \mathrm{~m}^{-2} \mathrm{~Hz}^{-1}$ )

contribution of $V_{Z}$ to the $V_{0}$ values calculated here is approximately $-0.1 V_{Z}$ and $0.2 V_{Z}$ for UFO2 and FLEETSAT respectively. After 22:00 LT, on magnetically quiet nights, vertical drift of the irregularities is basically that of the background plasma, and is much smaller than $V_{E}$ so that $V_{0} \approx V_{E}$. Therefore, an increase in $V_{0}$ with solar flux is in agreement with the observation by Fejer et al. (1991), based on Jicamarca incoherent scatter radar data from 1968 through 1989, that the nighttime eastward drift velocity of equatorial Fregion plasma increases with solar flux. As expected, smaller values of $V_{0}$ are obtained in the post-midnight period compared to the pre-midnight period when the height of the Flayer is greater.

In many earlier studies, the $50 \%$ decorrelation time for observed scintillations has been considered to be a measure of the Fresnel Scale, which requires $V_{0}$ to be constant and $V_{C}$ to be zero. Estimates of $V_{0}$ and $V_{C}$ from spaced receiver data have demonstrated that the above assumption does not hold. Rino and Owen (1980) found that under weak scatter conditions, wideband satellite signals recorded at the dip equatorial station Ancon in Peru, displayed a time structure with a coherence time, which was a monotonic function of the Fresnel radius divided by the effective scan velocity. However, the irregularity power spectral index determined the shape of this function. For weak scatter conditions, the coherence time or scale is independent of the irregularity strength. In the present study, use is made of the fact that $V_{C}$ has nonzero values, and variations in $V_{0}$ are automatically taken into account in the calculation of coherence scale $d_{I}$, so that these may be directly compared with results obtained in theoretical calculations by Engavale and Bhattacharyya (2005). These authors found that for a given average height of the irregularity layer and weak scintillations, $d_{I}$ increased with increasing value of the irregularity power spectral index. For a given irregularity power spectrum and weak scintillations, $d_{I}$ var-

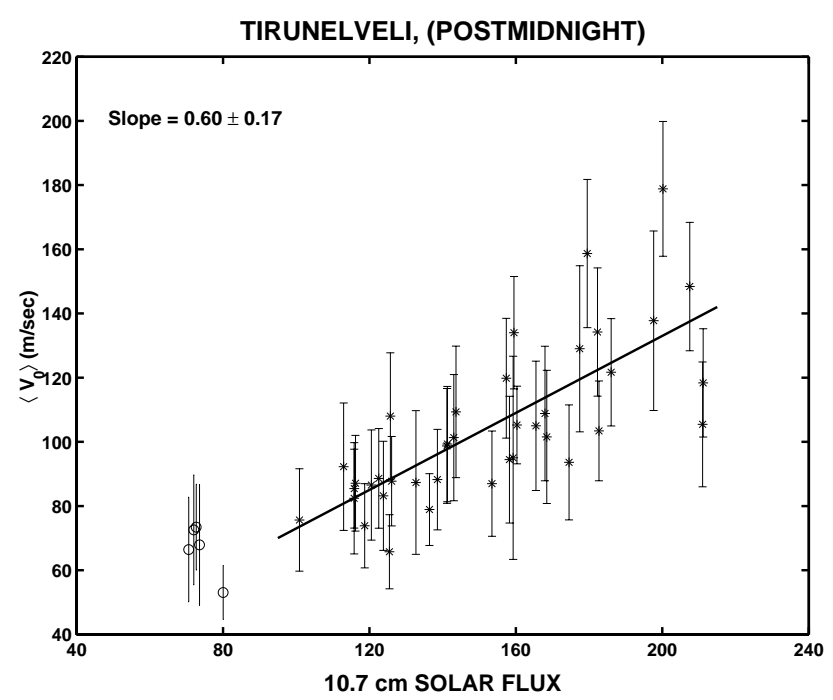

Fig. 9. plot of average $V_{0}$, for post-midnight hours versus monthly average 10.7-cm solar flux (in units of $10^{-22} \mathrm{~W} \mathrm{~m}^{-2} \mathrm{~Hz}^{-1}$ ).

ied approximately as the square root of the average height of the irregularity layer. Results for the post-midnight period depicted in Fig. 7 are not conclusive as regarding the dependence of $d_{I}$ on the 10.7-cm solar flux. Part of the scatter seen in the plots of $d_{I}$ versus solar flux may be attributed to the dependence of $d_{I}$ on the power spectral index of the irregularity spectrum (Engavale and Bhattachryya, 2005).

Figure 6, however, shows a clear tendency for premidnight $d_{I}$ values to increase with $10.7-\mathrm{cm}$ solar flux. It was shown by Fejer et al. (1999) that the threshold value of the vertical drift velocity for the generation of ESF irregularities increases with solar flux, which would imply that the postsunset irregularity layer is formed at a higher altitude as the solar flux increases. In a recent paper Jyoti et al. (2004) have demonstrated the existence of a threshold height of the base of the equatorial F-region, above which the polarity of meridional winds did not influence the occurrence of ESF irregularities, while below this threshold height triggering of ESF requires equatorward winds. This is expected, since with increasing altitude, as the ion-neutral collision frequency $v_{i n}$ decreases, contribution of the meridional wind to the linear growth rate of the Rayleigh-Taylor instability becomes less significant compared to $g /\left(v_{i n} L\right)$. Hence, the majority of the cases of ESF studied in the above paper (Jyoti et al., 2004) occurred when the threshold height was exceeded. These authors also found that the threshold height increases with the average 10.7-cm solar flux. Thus, the results shown in Fig. 6 imply that in the pre-midnight period, changes in $d_{I}$ are due to variations in the height of the post-sunset irregularity layer with $10.7 \mathrm{~cm}$ flux and that during the initial phase of ESF irregularity development, the irregularity spectrum is not as variable as in the post-midnight decay phase. 


\section{Conclusions}

Theoretical calculations have shown that during the period of weak scintillations, the dominant spatial scale in the ground scintillation pattern, given by the coherence scale length $d_{I}$, is determined by the average height of the irregularity layer and the irregularity power spectral index. Both of these factors are expected to be different for the pre- and postmidnight periods. Weak scintillations are generally expected to occur at the beginning and end of the scintillation event, which, respectively, occur when the ESF irregularities have just started to develop and during their decay phase. According to results obtained earlier (Fejer et al., 1999; Jyoti et al., 2004), the height of the ESF irregularities, which produce weak scintillations during the pre-midnight period, should show an increase with solar activity, as measured by the average 10.7-cm solar flux. Hence, it may be concluded from the pre-midnight results presented here for F $10.7-\mathrm{cm}$ solar flux exceeding 100, that in the initial development of ESF irregularities, the irregularity power spectrum shows little variation in the power spectral index. This behaviour is not seen during the post-midnight period, when no clear dependence of $d_{I}$ on the F 10.7-cm solar flux emerges, possibly due to much greater variability of the irregularity power spectral index caused by other factors which influence further evolution of the irregularities. In the future a more detailed study of this nature, using scintillation observations, may be used to understand the factors which influence the evolution of spatial structures in ESF irregularities after their initial development.

Acknowledgements. Topical Editor U.-P. Hoppe thanks two referees for their help in evaluating this paper.

\section{References}

Abdu, M. A., Medeiros, R. T., Bittencourt, J. A., and Batista, I. S.: Vertical ionization drift velocities and range type spread $\mathrm{F}$ in the evening equatorial ionosphere, J. Geophys. Res., 88, 399-402, 1983.

Bhattacharyya, A.: Role of E region conductivity in the development of equatorial ionospheric plasma bubbles, Geophys. Res. Lett., 31, L06806, doi:10.1029/2003GL018960, 2004.

Bhattacharyya, A., Basu, S., Groves, K. M., Valladares, C. E., and Sheehan, R. : Dynamics of equatorial F region irregularities from spaced receiver scintillation observations, Geophys. Res. Lett., 28, 119-122, 2001.

Bhattacharyya, A., Franke, S. J., and Yeh, K. C.: Characteristic velocity of equatorial F-region irregularities determined from spaced receiver scintillation data, J. Geophys. Res., 94, 1195911969, 1989.

Bhattacharyya, A., Groves, K. M., Basu, S., Kuenzler, H., Valladares, C., and Sheehan, R.: L-Band scintillation activity and space-time structure of low-latitude UHF scintillations, Radio Sci., 38(1), 1004, doi:10.1029/2002RS002711, 2003.
Brigg, B. H.: The analysis of spaced sensor records by correlation techniques, in: Middle Atmosphere program, Handbook for MAP, edited by: Vincent, R. A., Int. Counc. Of Sci. Unions, Paris, 13, 166-186, 1984.

Engavale, B. and Bhattacharyya. A.: Spatial correlation function of intensity variations in the ground scintillation pattern produced by equatorial spread-F irregularities, Indian J. Radio Space Phys., 34, 23-32, 2005.

Farley, D. T., Balsley B. B., Woodman R. F., and Mcclure, J. P.: Equatorial spread F: Implications of VHF radar observations, J. Geophys. Res., 75, 7199-7216, 1970.

Fejer, B. G.: Low latitude electrodynamic plasma drifts: A review, J. Atmos. Terr. Phys., 53, 677-693, 1991.

Fejer, B. G., Scherliess, L., and de Paula, E. R.: Effects of the vertical plasma drift velocity on the generation and evolution of Equatorial spread F, J. Geophys. Res., 104, 19859-19869, 1999.

Haerendel, G.: Theory of equatorial spread F, report, Max-PlanckInstitut fü extraterrestrische Physik, Garching, Germany, 1973.

Jayachandran, B., Balan, N., Rao, P. B., Sastri, J. H., and Bailey G. J.: HF Doppler and ionosonde observations on the onset conditions of equatorial spread F, J. Geophys. Res., 98, 13 741-13 750, 1993.

Jyoti, N., Devasia, C. V., Sridharan, R., and Tiwari, D.: Threshold height $\left(h^{\prime} F\right)_{c}$ for the meridional wind to play a deterministic role in the bottomside equatorial spread $\mathrm{F}$ and its dependence on solar activity, Geophys. Res. Lett., 31, L12809, doi:10.1029/2004GL019455, 2004.

Kelley, M. C. and Maruyama, T.: A diagnostic method for equatorial spread F, 2. The effect of magnetic activity, J. Geophys. Res., 97, 1271-1277, 1992.

Keskinen, M. J., Ossakow, S. L., Basu, S., and Sultan, P. J.: Magnetic-flux-tube-integrated evolution of equatorial plasma bubbles, J. Geophys. Res., 103, 3957-3967, 1998.

Keskinen, M. J., Ossakow, S. L., and Fejer, B. G.: Threedimensional nonlinear evolution of equatorial spread-F bubbles, Geophys. Res. Lett., 30(16), 1855, doi:10.1029/2003GL017418, 2003.

Ossakow, S. L.: Spread-F theories - a review, J. Atmos. Terr. Phys., 43, 437-452, 1981.

Rino, C. L. and Owen, J.: The time structure of transionospheric radio wave scintillation, Radio Sci., 15, 479-489, 1980.

Sastri, J. H.: Duration of equatorial spread F, Ann. Geophys., 2, 353-358, 1984.

Spatz, D. E., Franke, S. J., and Yeh, K. C.: Analysis and interpretation of spaced receiver scintillation data recorded at an equatorial station, Radio Sci., 23, 347-361, 1988.

Tsunoda, R. T.: Magnetic-field-aligned characteristics of plasma bubbles in the nighttime equatorial ionosphere, J. Atmos. Terr. Phys., 42, 743-752,1980.

Vacchione, J. D., Frank, S. J., and Yeh, K. C.: A new analysis technique for estimating zonal irregularity drifts and variability in the equatorial $F$ region using spaced receiver scintillation data, Radio Sci. 22, 745-756, 1987.

Wernik, A. W., Liu, C. H., and Yeh, K. C.: Modeling of spaced receiver scintillation measurements, Radio Sci, 18, 743-763, 1983.

Yeh, K. C. and Liu, C. H.: Radio-wave scintillations in the ionosphere, Proc. IEEE, 70, 324-360, 1982. 